\title{
Prolonged refeeding improves weight maintenance after weight loss with very-low-energy diets
}

\author{
Lena Gripeteg $^{1,2 *}$, Jarl Torgerson ${ }^{1,3}$, Jan Karlsson ${ }^{4}$ and Anna Karin Lindroos ${ }^{5}$ \\ ${ }^{1}$ Department of Molecular and Clinical Medicine, Institute of Medicine, The Sahlgrenska Academy, University of Gothenburg, \\ Gothenburg, Sweden \\ ${ }^{2}$ Vårdalinstitutet, Lund, Sweden \\ ${ }^{3}$ Department of Health Care, Regional Secretariat, Gothenburg, Sweden \\ ${ }^{4}$ Institute of Health and Care Sciences, The Sahlgrenska Academy, University of Gothenburg, Gothenburg, Sweden \\ ${ }^{5}$ MRC Human Nutrition Research, Cambridge, UK \\ (Received 19 November 2008 - Revised 5 June 2009 - Accepted 8 June 2009 - First published online 7 August 2009)
}

The aim of the present study was to test the hypothesis that a prolonged refeeding duration after successful very-low-energy diet (VLED)-induced weight loss beneficially affects weight development and eating behaviour. Patients ( $n$ 269) were recruited to a 1-year obesity treatment programme with 12 weeks of an initial VLED. After the VLED, patients with $\geq 10 \%$ weight loss were randomly allocated to 1 week (group 1 ) or 6 weeks (group 6) refeeding to an ordinary, energy-reduced diet, and thereafter followed and actively treated for an additional 40 weeks. Eating behaviour (revised twenty-one-item Three-Factor Eating Questionnaire) was measured at baseline, during and after refeeding, and at week 52. Weight change over time in the two treatment groups was tested by repeated-measures analysis in completers and by intention to treat (ITT). Of the patients, 169 (109 women) lost $\geq 10 \%$ during the VLED and were randomised. At randomisation, weight loss was - 16.5 (SD 3.7) \% in group 1 and -16.7 (SD 4.3) \% in group $6(P=0.73)$. Between weeks 12 and 52, completers in group 6 regained significantly less weight (3.9 (SD 9.1) \%) as compared with group 1 (8.2 (SD 8.3) \%; $P=0.006)$ (ITT, $P=0.05)$. Completers in group 6 also maintained a higher level of dietary restraint after refeeding was completed, but eating behaviour did not differ at week 52 . Weight change after the refeeding periods were completed did not differ significantly between the groups $(P=0 \cdot 06)$. Overall, longer refeeding duration after successful weight loss with a VLED improves weight maintenance in a 1-year perspective.

\section{Obesity: Randomised trials: Very-low-energy diets: Three-Factor Eating Questionnaire}

Very-low-energy diets (VLED) can be used to accomplish rapid and substantial weight reduction in obese patients ${ }^{(1)}$. VLED are liquid formulas containing all essential nutrients with an energy content of $1 \cdot 9-3 \cdot 3 \mathrm{MJ} / \mathrm{d}^{(2)}$. The low energy level results in weight losses of $1.5-2 \cdot 5 \mathrm{~kg} / \mathrm{week}^{(2)}$ with concurrent risk factor improvements ${ }^{(3,4)}$.

During a VLED food choice is easy since energy-free fluid is the only complement needed. Also, it has been suggested that food cravings are more reduced with VLED than with food-based low-energy diets ${ }^{(5)}$, which may contribute to better dietary adherence ${ }^{(6)}$. Studies have shown that greater early weight reduction ${ }^{(7-10)}$ and the use of $\operatorname{VLED}^{(8)}$ predict greater weight loss long term. However, after a VLED period, gradual weight rebound is generally seen ${ }^{(11-13)}$. Alternative strategies have been suggested to prevent or limit weight regain after a VLED. Different supplementations do not seem to have an effect on post-VLED weight development $^{(14-16)}$, but improved weight maintenance has been observed with pharmacological therapy ${ }^{(17,18)}$, exercise ${ }^{(19)}$, continued use of VLED as part of the dietary allowance ${ }^{(20)}$ and protein supplementation ${ }^{(21)}$.
An important phase in VLED programmes is when ordinary foods are reintroduced. Throughout refeeding, patients are required to adopt strategies to adjust eating behaviour and dietary intake to the lower energy requirements of a reduced body weight. Thus, the duration of refeeding may have an impact on the ability to achieve long-term weight control. Refeeding periods of 1 to 6 weeks ${ }^{(5,13,18,20,22-27)}$ have been reported, but the long-term effect of different refeeding strategies has not been examined. Therefore, our objective was to test the hypothesis that prolonged refeeding duration after successful VLED-induced weight loss beneficially affects weight development and eating behaviour in a 1-year perspective.

\section{Experimental methods}

Study design and subjects

A non-blinded, randomised, 1-year clinical trial with parallel treatment groups was conducted to examine the effect of prolonged refeeding on weight maintenance and eating behaviour in obese patients after a VLED-induced weight loss. 
Patients referred to the Obesity Unit at Sahlgrenska University Hospital were eligible if aged 18-60 years, BMI $>30.0 \mathrm{~kg} / \mathrm{m}^{2}$ and Swedish speaking. Subjects were not eligible if contraindications for VLED treatment were identified at an initial medical and dietary examination. Contraindications included pregnancy and lactation, unstable type 1 diabetes or cardiac disease, recent cerebrovascular disease, a history of eating disorder, severe psychiatric disorder or other severe disease. Weight loss medication was not allowed during the trial. All patients were recruited to participate in a 1-year structured obesity intervention with 12 initial weeks of VLED. The study was approved by the regional ethical review board and registered with ClinicalTrials.gov (no. NCT00694811; http://www.clinicaltrials.gov). All participants gave written informed consent.

In total, 300 patients were enrolled between August 2004 and January 2007 (Fig. 1). Of these, thirty-one withdrew from the study before treatment started. Thus, 269 patients started the VLED phase of the study and 169 were subsequently randomised after 12 weeks to 1 or 6 refeeding weeks, and followed and actively treated for an additional 40 weeks. The weight loss criterion for randomisation was $\geq 10 \%$ over the 12 -week VLED period. This cut-off was chosen as studying optimal refeeding time after VLED is only relevant for subjects who comply with their VLED. Non-randomised patients were offered routine treatment at the obesity unit.

\section{Very-low-energy diet programme}

During the 12 initial weeks, patients were encouraged to follow a strict liquid VLED $(2 \cdot 0-3 \cdot 4 \mathrm{MJ} / \mathrm{d})$. Patients selected and paid themselves (total cost 5-7 euro/d) for one of the following commercially available diets: Modifast ${ }^{\circledR}(3.4 \mathrm{MJ} / \mathrm{d}$; Novartis, Veenendaal, The Netherlands), Nutrilett ${ }^{\circledR} \quad(2.3 \mathrm{MJ} / \mathrm{d}$; Axellus AB, Solna, Sweden) or Cambridgekuren ${ }^{\circledR}(2.0 \mathrm{MJ} / \mathrm{d}$; Cambridgekuren, Solna, Sweden). Free consumption of nonenergy beverages $(<25 \mathrm{~kJ} / 100 \mathrm{~g})$ was allowed. All patients had scheduled nurse visits at weeks 0 (baseline), 2, 5, 8 and 12. Body weight was registered at each visit and patients were given support and counselling to enhance VLED compliance. Side effects were monitored and more frequent contacts were offered if needed. All patients were encouraged to be physically active.

\section{Dietary treatment}

Following VLED, dietary treatment advice was given during nine individual sessions with a dietitian (weeks 12, 15, 18, 21, 26, 32, 38, 44 and 52). At week 12 (the first dietitian visit), the dietitian assigned patients with $\geq 10 \%$ weight loss to treatment group according to randomisation. Standardised written and oral instructions for 1 or 6 weeks refeeding were provided; hence, participants as well as study staff were aware of group assignment. During refeeding, ordinary meals

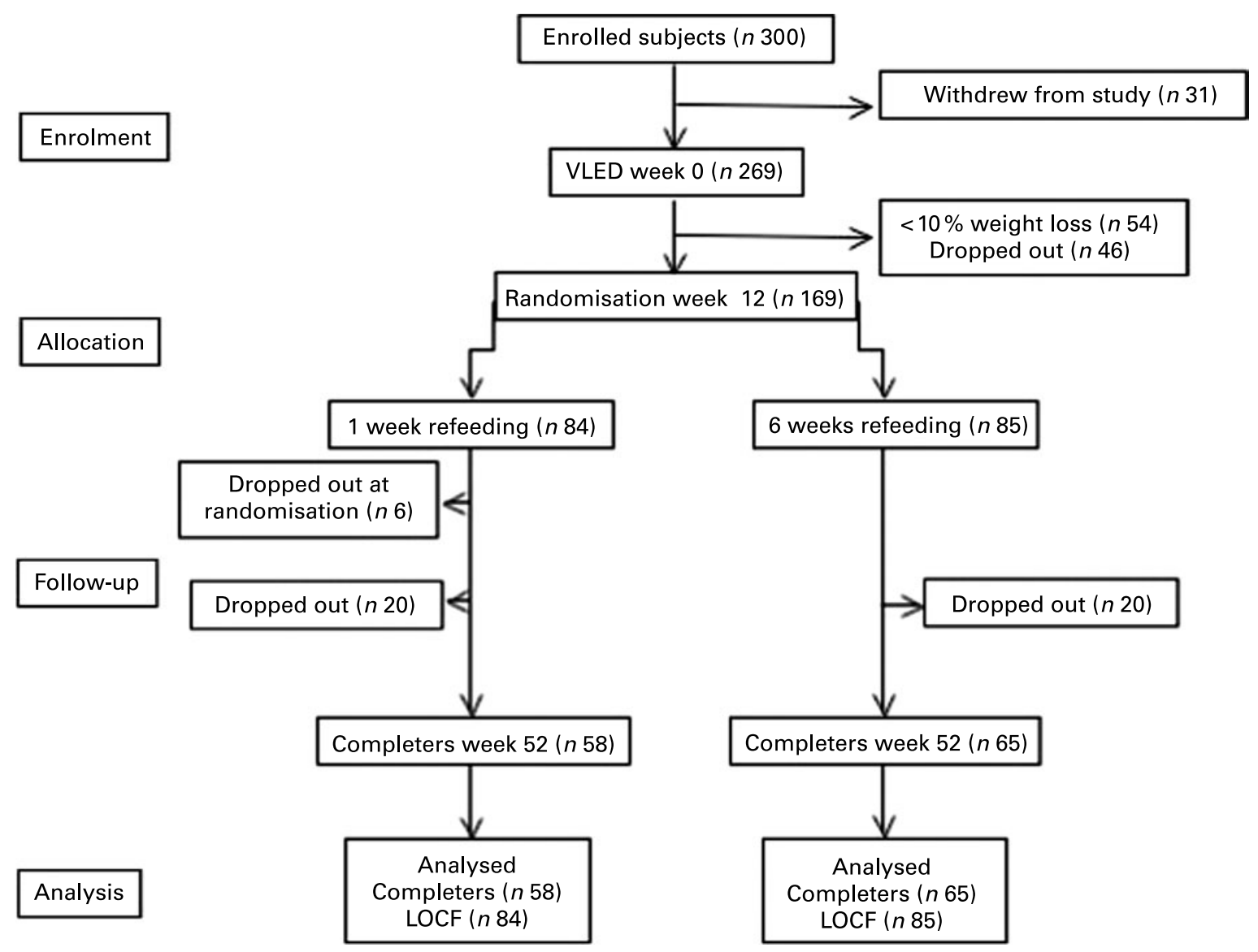

Fig. 1. Flow chart of the study. VLED, very-low-energy diet; LOCF, last observation carried forward. 
were gradually reintroduced and VLED portions removed. Patients with 1 week refeeding (group 1) started with breakfast for $3 \mathrm{~d}$, added lunch or dinner the next $4 \mathrm{~d}$, and thereafter the third meal was reintroduced and the VLED discontinued. Patients with 6 weeks refeeding (group 6) started with one regular meal (patient's choice) for 3 weeks. A second meal was added the next 3 weeks, and at week 7 the third meal was reintroduced and the VLED discontinued. Low-energy between-meal snacks (for example, fruit) were allowed from week 14 for group 1 and week 15 for group 6 .

Recommended energy intake was based on estimated energy requirement minus $30 \%$ to achieve an energy-reduced level. Energy requirement was calculated from the equation of Harris-Benedict ${ }^{(28)}$ for estimation of BMR, and multiplied by a factor of 1.3 for total energy expenditure (moderate physical activity level). Recommended energy distribution was for breakfast $20 \%$, lunch $35 \%$, and dinner $35 \%$ of daily energy intake, and $10 \%$ from snacks. Dietary prescriptions followed Swedish Nutrition Recommendations ${ }^{(29)}$.

All patients received written dietary advice based on three main meals with portion sizes in grams of different meal components. The purpose of the dietary treatment was to provide each patient with tailored counselling based on individual needs in order to maintain achieved weight loss. The treatment focused on long-term behavioural change and self-monitoring tasks were used. The specific dietary advice covered meal pattern and composition, food choice, portion size, eating behaviour and physical activity. With the exception of refeeding strategies, both groups received the same treatment and advice.

\section{Measurements}

Body weight was measured at all visits, in underwear and without shoes to the nearest $0.1 \mathrm{~kg}$ using electronic scales. Height was measured at baseline in the standing position, without shoes, to the nearest $0.5 \mathrm{~cm}$. BMI was calculated $\left(\mathrm{kg} / \mathrm{m}^{2}\right)$.

At baseline and weeks 26 and 52, body circumferences and blood pressure were measured and overnight fasting blood samples drawn. Waist circumference was measured midway between the costal arch and the iliac crest and hip circumference at the symphysis-trochanter femoris level to the nearest $1.0 \mathrm{~cm}$. At baseline, systolic and diastolic blood pressure was measured once in both arms, after which the arm with the highest pressure was used throughout the study. At weeks 26 and 52, systolic and diastolic blood pressure was measured twice in the sitting position after 5 min rest, registering the mean value. All measurements were performed with calibrated equipment by trained staff. Blood chemistry included fasting plasma glucose, serum HDL-cholesterol and serum TAG. Serum insulin was measured at baseline.

The following cut-offs for cardiovascular risk factors were used: hypertension, systolic and diastolic blood pressure $\geq 135$ / $85 \mathrm{mmHg}$; hyperglycaemia, plasma glucose $\geq 6.1 \mathrm{mmol} / \mathrm{l}$; low HDL-cholesterol, serum HDL $<1.3$ and $1.0 \mathrm{mmol} / 1$ for women and men; hypertriacylglycerolaemia, serum TAG $\geq 1.7 \mathrm{mmol} / \mathrm{l}$; central obesity, waist circumference $>88$ and $>102 \mathrm{~cm}$ for women and men, respectively ${ }^{(30)}$.

Self-assessed eating behaviour was measured at baseline and weeks 15, 21, 26 and 52, using the revised 21-item
Three-Factor Eating Questionnaire, which covers three eating behaviour domains: cognitive restraint, emotional eating and uncontrolled eating ${ }^{(31,32)}$.

\section{Power calculation and randomisation}

Sample sizes for recruitment and randomisation were calculated using information from a similar study ${ }^{(11)}$. Based on $80 \%$ power to detect a $4 \mathrm{~kg}$ weight difference between the groups, standard deviation $7 \mathrm{~kg}$, statistical significance level $P<0.05$, and $35 \%$ drop-out between weeks 12 and 52, a sample size of seventy-five patients per treatment arm was required for randomisation (week 12). As some patients were expected to drop out from the VLED phase of the study and others not to lose enough weight, a total of 300 patients were recruited to the study.

Patients were eligible for randomisation if they had lost $\geq 10 \%$ of the initial body weight after 12 VLED weeks. The randomisation was stratified by sex and degree of weight loss (strictly greater than or less than 17.1\%). Eligible patients were randomly assigned within each of the four strata to one of the two treatments ( 1 or 6 weeks refeeding) in blocks of size two, with equal allocation of treatments within each block. The randomisation list was generated with a pseudo-random number generator. The treatment allocation order was arranged in numbered, sealed envelopes and kept in separate boxes for each stratum. At patient assignment, the next envelope in order was drawn from the appropriate box.

\section{Analysis and statistics}

The primary outcome measure was the difference in weight change $(\%)$ from week 12 to 52 between the two treatment groups. Secondary outcome measures were changes in eating behaviour scores (Three-Factor Eating Questionnaire) and cardiovascular risk factors at week 52 as compared with baseline. As the refeeding periods differed between the groups ( 1 week in group 1 and 6 weeks in group 6), a post hoc analysis was also carried out to test whether weight change differed between the two groups after the refeeding periods were completed. In this analysis time-points for baseline and endpoint thus differed between the groups with baseline and endpoint at weeks 13 and 47 in group 1, and at weeks 18 and 52 in group 6. The weight values at week 13 and at week 47 were imputed from the weights measured at the closest time points before and after weeks 13 and 47, respectively. Results are reported as mean values and standard deviations or as frequencies if not otherwise stated. Differences between groups were tested by the two-sample $t$ test (parametric variables) or the Wilcoxon two-sample test (non-parametric variables). Changes within groups were tested by the paired $t$ test. The $\chi^{2}$ test was used to compare dichotomous data. Baseline to week 52 weight changes in the two treatment groups were tested by repeated-measures analysis in completers, and on an intention-to-treat basis with the last weight observation carried forward. In the post hoc analysis, analysis of covariance was used to test the difference in weight change from the new baselines (weeks 13 and 18) to the new endpoints (weeks 47 and 52), with weight loss $(\%)$ from study start (before the VLED) to the 
new baselines as a covariate. Statistical significance was set at two-tailed $P<0.05$. All statistical analyses were performed using SAS-PC software (version 9.1; SAS Institute Inc., Cary, NC, USA).

\section{Results}

\section{Participants}

After 12 weeks of the VLED, 169 patients met the randomisation criteria (Fig. 1). Baseline characteristics for patients assigned to 1 (group 1, $n$ 84) or 6 weeks (group 6, $n$ 85) refeeding are shown in Table 1 . Of the patients, $64 \%$ were women. Sex distribution, age, anthropometry or cardiovascular risk factors at baseline did not differ between the groups.

\section{Body weight}

Figure 2 shows relative weight change over 1 year for the 123 completers by treatment group. At randomisation, mean weight loss was -16.5 (SD 3.7) $\%$ in group 1 and -16.7 (SD 4.3$) \%$ in group $6(P=0.73)$. Between weeks 12 and 52, weight increased by 8.2 (SD 8.3 ) $\%$ in group 1 and 3.9 (SD 9.1 ) $\%$ in group 6. Group 6 regained significantly less weight over time, both in a completers' analysis $(P=0.006)$ and in an intention-to-treat analysis with last observation carried forward $(P=0 \cdot 05)$. Furthermore, the overall weight loss at week 52 compared with baseline was significantly greater in group $6(-13.4(\mathrm{SD} 8 \cdot 4) \%)$ than in group $1(-10 \cdot 3(\mathrm{SD} 7 \cdot 5)$ $\%)(P=0.03)$. Of patients allocated to group 1 and group 6, thirty-one $(37 \%)$ and forty-one $(48 \%)$, respectively, had achieved a weight reduction of $\geq 10 \%$ at week 52 $(P=0 \cdot 13)$. Eight $(10 \%)$ and twenty $(24 \%)$ patients in group 1 and group 6 , respectively, continued to lose weight

Table 1. Patient characteristics at baseline and body weight at randomisation*

(Mean values and standard deviations)

\begin{tabular}{|c|c|c|c|c|}
\hline & \multicolumn{2}{|c|}{ Group 1 ( $n$ 84) } & \multicolumn{2}{|c|}{ Group 6 (n 85) } \\
\hline & Mean & SD & Mean & SD \\
\hline \multicolumn{5}{|l|}{ Subjects $(n)$} \\
\hline Female & \multicolumn{2}{|c|}{54} & \multicolumn{2}{|c|}{55} \\
\hline Male & \multicolumn{2}{|c|}{30} & \multicolumn{2}{|c|}{30} \\
\hline Age (years) & $40 \cdot 6$ & $9 \cdot 2$ & $41 \cdot 9$ & $10 \cdot 2$ \\
\hline Height (m) & $1 \cdot 72$ & 0.1 & $1 \cdot 72$ & 0.1 \\
\hline Weight at baseline $(\mathrm{kg})$ & $122 \cdot 9$ & $23 \cdot 0$ & $124 \cdot 6$ & $25 \cdot 8$ \\
\hline Weight at randomisation $(\mathrm{kg}) \dagger$ & $102 \cdot 8$ & $20 \cdot 7$ & $104 \cdot 0$ & $23 \cdot 0$ \\
\hline BMI at baseline $\left(\mathrm{kg} / \mathrm{m}^{2}\right)$ & 41.4 & $5 \cdot 7$ & $41 \cdot 7$ & $6 \cdot 3$ \\
\hline Waist circumference $(\mathrm{cm})$ & $124 \cdot 8 \ddagger$ & $14 \cdot 8$ & $125 \cdot 5 \S$ & $15 \cdot 9$ \\
\hline Systolic blood pressure $(\mathrm{mmHg})$ & $128 \cdot 8$ & $16 \cdot 4$ & $130 \cdot 8$ & $16 \cdot 5$ \\
\hline Diastolic blood pressure $(\mathrm{mmHg})$ & $84 \cdot 0$ & $10 \cdot 3$ & $85 \cdot 7$ & $12 \cdot 7$ \\
\hline Serum insulin (mU/l) & $21 \cdot 7 \ddagger$ & $14 \cdot 3$ & $25 \cdot 2$ & $25 \cdot 4$ \\
\hline Plasma glucose (mmol/l) & $5 \cdot 5$ & $1 \cdot 3$ & $5 \cdot 4 \ddagger$ & $1 \cdot 3$ \\
\hline Serum HDL-cholesterol (mmol/l) & $1 \cdot 3 \ddagger$ & $0 \cdot 2$ & $1 \cdot 3 \S$ & 0.3 \\
\hline Serum TAG (mmol/l) & $1 \cdot 6 \ddagger$ & 0.8 & $1.5 \S$ & 0.7 \\
\hline
\end{tabular}

Group 1, 1 week refeeding, group 6, 6 weeks refeeding.

${ }^{*}$ For details of subjects and procedures, see Experimental methods. No significant differences were observed between the groups.

$\dagger$ Measured at week 12 (after very-low-energy diet).

$\ddagger n 83$.

$\S n 84$. after ordinary foods were reintroduced and had a lower weight at week 52 than at week $12(P=0 \cdot 01)$.

To remove the effect of different refeeding periods between the groups, 34-week weight change after the refeeding periods were completed was also calculated. The weight change was 6.9 (SD 7.6) \% between weeks 13 and 47 in group 1, and 4.3 (SD 7.5) \% between weeks 18 and 52 in group 6. When controlling for weight change from study start to the end of the respective refeeding periods the difference between the groups was not statistically significant $(P=0.06)$.

\section{Eating behaviour}

Baseline eating behaviour did not differ between the two treatment groups (Table 2). Substantial changes were observed in both groups after the VLED period, i.e. cognitive restraint increased, while uncontrolled and emotional eating decreased. All changes were statistically significant, except for emotional eating at week 52. At weeks $21(P=0.01)$ and $26(P=0.02)$, dietary restraint was significantly higher in group 6 than in group 1. After 1 year, cognitive restraint and uncontrolled eating had significantly improved in both groups as compared with baseline, while emotional eating did not differ from baseline values. The changes in eating behaviour at week 52 as compared with baseline did not differ significantly between treatment groups.

\section{Cardiovascular risk factors}

At week 52, waist circumference, systolic and diastolic blood pressure, plasma glucose, serum HDL and serum TAG were significantly improved in both treatment groups as compared with baseline, with the exception of diastolic blood pressure in group 1 (Fig. 3). However, there were no significant differences between groups at week 52 . From baseline to week 52, the number of patients with

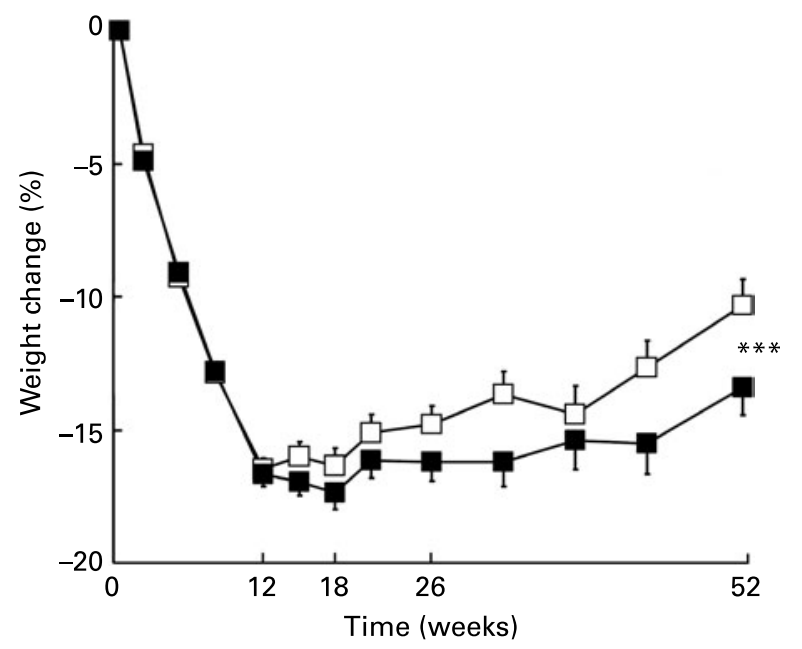

Fig. 2. Changes (\%) in weight among completers during 1 treatment year with 12 initial weeks of very-low-energy diet followed by 1 (group 1; $\square$ ) or 6 weeks (group $6 ; \mathbf{\square}$ ) refeeding to an ordinary, energy-reduced diet. Values are means, with standard errors represented by vertical bars. ${ }^{* \star *}$ Significant difference between groups over time in completers' analysis $(P=0.006)$ and in intention-to-treat analysis ( $P=0.05$; repeated-measures analysis). 
Table 2. Self-assessed eating behaviour (revised twenty-one-item Three-Factor Eating Questionnaire) before and after weight loss with very-low-energy diets $(\mathrm{VLED})^{*} \dagger$

(Mean values and standard deviations)

\begin{tabular}{|c|c|c|c|c|c|c|c|c|c|c|}
\hline \multirow[t]{2}{*}{ Time... } & \multicolumn{2}{|c|}{ Week 0} & \multicolumn{2}{|c|}{ Week 15} & \multicolumn{2}{|c|}{ Week 21} & \multicolumn{2}{|c|}{ Week 26} & \multicolumn{2}{|c|}{ Week 52} \\
\hline & Mean & SD & Mean & SD & Mean & SD & Mean & SD & Mean & SD \\
\hline \multicolumn{11}{|c|}{ Subjects $(n)$} \\
\hline Group 1 & \multicolumn{2}{|c|}{82} & \multicolumn{2}{|c|}{63} & \multicolumn{2}{|c|}{57} & \multicolumn{2}{|c|}{58} & \multicolumn{2}{|c|}{53} \\
\hline Group 6 & \multicolumn{2}{|c|}{83} & \multicolumn{2}{|c|}{72} & & & \multicolumn{2}{|c|}{73} & \multicolumn{2}{|c|}{62} \\
\hline \multicolumn{11}{|c|}{ Cognitive restraint } \\
\hline Group 1 & 41.9 & $17 \cdot 5$ & $65 \cdot 3$ & $15 \cdot 0$ & $61 \cdot 8$ & $15 \cdot 5$ & 59.5 & $17 \cdot 4$ & $56 \cdot 4$ & $20 \cdot 6$ \\
\hline Group 6 & $43 \cdot 0$ & $20 \cdot 9$ & $67 \cdot 6$ & $17 \cdot 4$ & $67.9 \ddagger$ & $17 \cdot 2$ & $66.0 \ddagger$ & $15 \cdot 2$ & $59 \cdot 4$ & $18 \cdot 3$ \\
\hline \multicolumn{11}{|c|}{ Uncontrolled eating } \\
\hline Group 1 & 44.5 & $19 \cdot 2$ & 28.0 & $17 \cdot 1$ & $32 \cdot 0$ & $17 \cdot 0$ & $30 \cdot 4$ & $19 \cdot 2$ & 38.0 & $24 \cdot 6$ \\
\hline Group 6 & $45 \cdot 0$ & $22 \cdot 1$ & 29.9 & $19 \cdot 6$ & 33.0 & $20 \cdot 8$ & $32 \cdot 3$ & 20.5 & 35.5 & $23 \cdot 3$ \\
\hline \multicolumn{11}{|c|}{ Emotional eating } \\
\hline Group 1 & $54 \cdot 3$ & $28 \cdot 7$ & $37 \cdot 1$ & $23 \cdot 2$ & 40.5 & 24.5 & $42 \cdot 6$ & $26 \cdot 8$ & $46 \cdot 6$ & 28.8 \\
\hline Group 6 & $50 \cdot 6$ & $28 \cdot 3$ & $33 \cdot 1$ & $23 \cdot 1$ & $40 \cdot 9$ & $28 \cdot 7$ & $42 \cdot 5$ & $28 \cdot 7$ & 44.9 & 31.4 \\
\hline
\end{tabular}

Group 1, 1 week refeeding, group 6, 6 weeks refeeding.

${ }^{*}$ Within the two groups, all mean values were significantly different from those at baseline $(P<0.01)$, with the exception of emotional eating at week 52 (NS).

† Scale range is $0-100$. Higher scores represent more restraint, uncontrolled, and emotional eating. Measured at week 0 (before VLED) and at 3 (study week 15), 9 (study week 21), 14 (study week 26) and 40 (study week 52) weeks after randomisation (week 12 , end of VLED).

$\ddagger$ Mean value was significantly different from that of group $1(P<0.05)$.

three or more risk factors, the Adult Treatment Panel III criteria for the metabolic syndrome ${ }^{(30)}$, decreased from $26 \%$ to $10 \%(P<0.0001)$.

\section{Drop-out and adverse events}

Six patients allocated to group 1 dropped out at randomisation and twenty subjects from each group dropped out during the post-VLED dietary intervention (Fig. 1). The entire treatment programme was completed by thirty-seven of fifty-four women and twenty-one of thirty men in group 1 and by forty of fifty-five women and twenty-five of thirty men in group 6. Drop-out rate did not differ by sex or by treatment arm. In total, fifty-eight $(69 \%)$ and sixty-five $(76 \%)$ of participants in group 1 and group 6 completed the entire study $(P=0 \cdot 28)$.

In general, the refeeding period and the following dietary treatment were well tolerated. Three women in each group became pregnant and were therefore excluded. One woman in group 1 died of heart failure at week 35 .
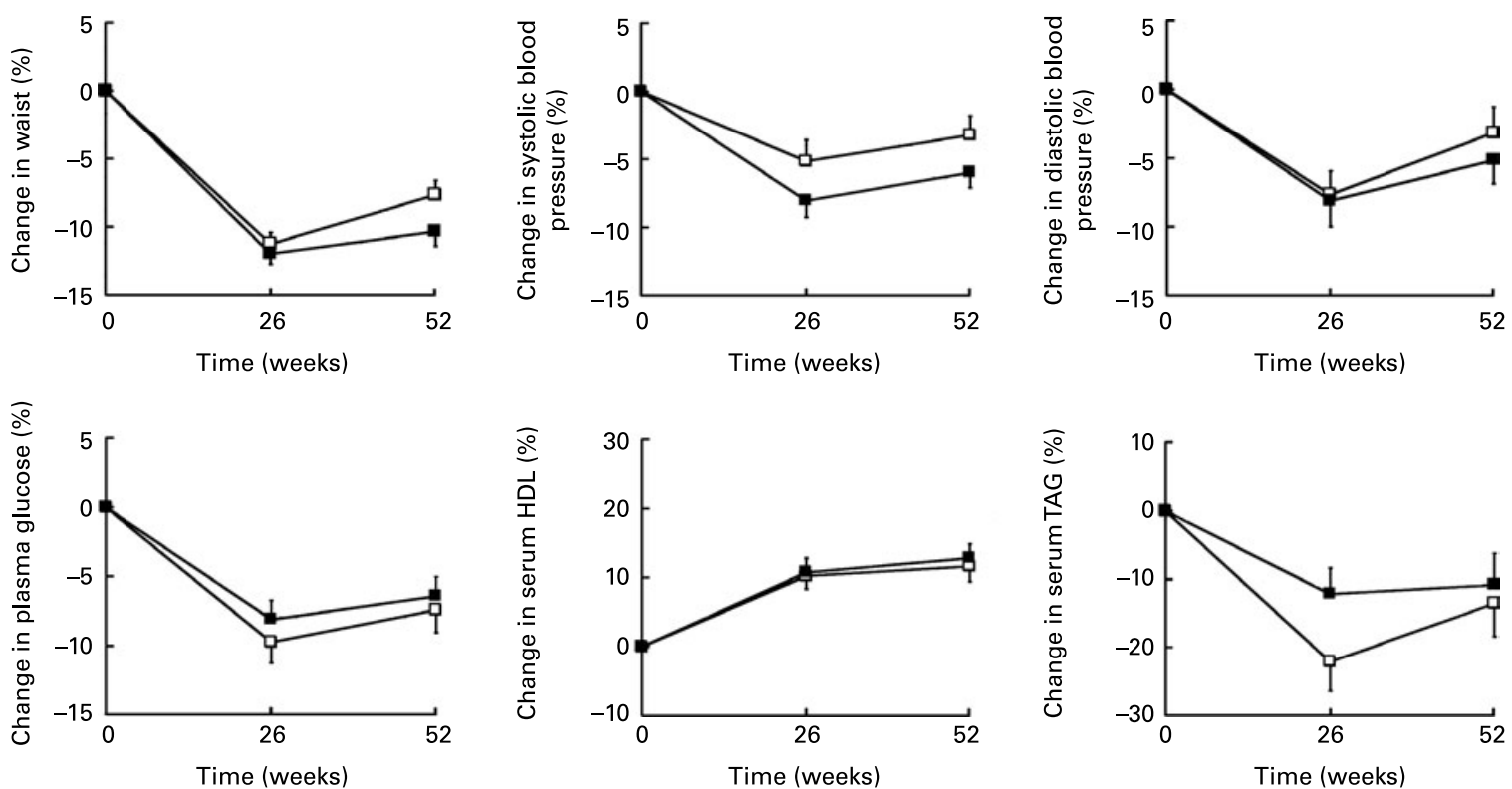

Fig. 3. Changes (\%) in risk factors after 26 and 52 weeks of treatment. ( $\square$ ), Refeeding for 1 week to an ordinary, energy-reduced diet (group 1 ); ( $\square$ ), refeeding for 6 weeks to an ordinary, energy-reduced diet (group 6). Values are means, with standard errors represented by vertical bars. All risk factors were significantly improved in both treatment groups at week 52, except for diastolic blood pressure in group 1. No significant differences were found between treatment groups at week 52. 


\section{Discussion}

The focus of the present study was to evaluate the impact of refeeding duration on post-VLED weight development in obese patients referred to an obesity clinic. The present results show better 1-year weight loss with a longer refeeding period of 6 weeks compared with a shorter refeeding period of 1 week.

It has previously been shown that strict VLED adherence is better than a liberal VLED regimen ${ }^{(23)}$, and that the incorporation of VLED in the meal plan after the weight loss period improves long-term weight maintenance ${ }^{(20)}$. To our knowledge, this is the first study that has evaluated the effects of refeeding on weight regain. Consistent with our main hypothesis, patients with a prolonged, 6-week, refeeding period lost significantly more weight over 1 year of treatment than the group with 1-week refeeding. Weight loss after the refeeding periods were completed did not differ significantly between the groups, although the $P$ value was close to 0.05 in this post hoc analysis. This suggests that the better weight maintenance in group 6 was to some extent explained by continued weight loss or better weight maintenance during the longer refeeding period. Recommended energy intake was lower in group 6 during the longer refeeding period. For patients recommended $6 \cdot 3 \mathrm{MJ} / \mathrm{d}$, the energy deficit over the first 6 weeks after the VLED was about 54.4 MJ more for patients on 6 weeks refeeding compared with patients on 1 week refeeding. Although this corresponds to approximately $2 \mathrm{~kg}$ of fat mass, the calculation is entirely theoretical as it assumes $100 \%$ compliance to the recommended meal plans. Based on our clinical experience this is very unlikely and efforts related to improving patients' compliance to a low energy intake is essential for long-term weight maintenance. We hypothesised that a slower reintroduction of food after the VLED would improve the patients' eating behaviour, as they could focus on one meal at a time and get longer time to practise and adjust to each meal. In line with this hypothesis the group with the longer refeeding period maintained higher levels of dietary restraint after the refeeding was completed, reflecting an improved ability to restrict food intake and follow dietary prescriptions.

The observed changes in eating behaviour are in accordance with previous studies describing an increase in restrained eating $^{(19,33,34)}$ and a decrease in uncontrolled and emotional eating ${ }^{(19,34)}$ during weight loss. This overall pattern of eating behaviour changes has been associated with favourable changes in dietary intake ${ }^{(34)}$. Also, high scores in cognitive restraint have been associated with weight loss and adaptation of weight-controlling behaviour ${ }^{(33)}$. Although eating behaviour tended to return towards baseline level over time, cognitive restraint and uncontrolled eating were still significantly improved in both treatment groups after 1 year.

As previously observed, 1 year weight losses in the order of $10 \%$ were related to significant improvements in cardiovascular risk factors ${ }^{(11)}$. In group 6 , improvements in the range of $10 \%$ were seen for blood lipids and waist circumference, whereas the improvements for plasma glucose and blood pressure were about $5 \%$. The improvements were slightly smaller in group 1 for most of the risk factors, but the differences were not statistically significant.

It should be noted that the patients included in the present study are obese patients referred to a specialist obesity clinic by their general practitioners. Average weight was therefore higher and co-morbidities more prevalent than in most VLED studies. This may explain why not all patients reached the randomisation phase of the study $(17 \%$ of the patients dropped out during the VLED period and $20 \%$ did not lose enough weight). We chose to randomise patients with weight losses greater than $10 \%$ after VLED. Thus, only successful patients were included. This is a strategy used in other studies ${ }^{(18)}$ and it could be argued that refeeding is relevant only when a substantial VLED-induced weight loss has actually been achieved. After 1 year, $43 \%$ of the 169 randomised patients maintained a weight reduction of at least $10 \%$, whereas $17 \%$ continued to lose weight after the VLED period and had a greater weight loss at week 52 than after the 12-week VLED.

A total of $27 \%$ of the patients dropped out during the 40 weeks of treatment after the VLED. Similar attrition rates have been reported from other randomised VLED programmes ${ }^{(20,23)}$. However, it should be noticed that six women were excluded due to pregnancy and should therefore not be regarded as actual drop-outs. Increased fertility in obese women who lose weight has also been observed in other studies $^{(20,35)}$. A difficulty in most dietary interventions is that patients and study staff cannot be blinded to treatment allocation. Although all patients agreed to the study protocol at baseline, six patients from group 1 chose to drop out at randomisation while no patients from group 6 left the trial at randomisation. This implies that some participants considered 1 week refeeding as a less advantageous alternative. Nevertheless, long-term drop-out was similar in the two treatment groups. In the present study, patients were followed for 40 weeks, which is a relatively short time period considering that obesity is a chronic disorder. However, although a longer follow-up is desirable, we believe that the achieved 1-year improvements in body weight and risk factors are clinically relevant and the present results contribute to a better understanding of how to improve weight maintenance after VLED in obesity treatment programmes.

A difficulty, complicating the interpretation of the present study, is that food was reintroduced over different time periods resulting in different energy recommendations during the first 6 weeks of the weight-maintenance phase. More frequent weight measurements and information on food intake during the refeeding phase of the study would have allowed us to explore in more detail how recommended energy intake and approach to food introduction influenced actual energy intake and eating behaviour. However, for practical reasons we could not fit in more visits in the treatment programme and in this group of obese patients we considered food records too extensive for both patients and staff. Furthermore, we doubted that we would be able to get reliable information on dietary intake ${ }^{(36)}$. In order to take the different finishing moments in time into account, we investigated the difference in weight change from the end of the refeeding periods (week 13 and 18) to weeks 47 and 52, respectively, using imputed weight measurements in group 1. This post hoc analysis adds to the overall picture, but introduces a new time imbalance as the overall, active treatment time was 5 weeks shorter in group 1 compared with group 6 .

In conclusion, after successful VLED-induced weight loss, patients with 6 weeks refeeding maintained a significantly 
greater weight loss over 1 year of treatment than patients with only 1 -week refeeding due to a significantly smaller postVLED weight regain. The beneficial effect of the longer refeeding period on weight maintenance was to some extent due to keeping the VLED as part of the diet for an extended time, but also due to higher levels of dietary restraint after the refeeding period. Accordingly, the present study implies that ordinary foods should be reintroduced slowly after a VLED period to enhance weight control. In addition to dietary counselling and support, the prolonged refeeding could be combined with other post-VLED approaches, such as a highprotein diet ${ }^{(21)}$, physical activity ${ }^{(19)}$ and/or pharmacological therapy ${ }^{(17,18)}$, to further improve long-term treatment outcome.

\section{Acknowledgements}

We want to thank the staff at the Obesity Unit at Sahlgrenska University Hospital for their committed work with the present study. We also thank Dr Markku Peltonen for statistical advice and Karin Lantz and Margareta Rystedt for excellent assistance with collating data.

The present study was supported by grants from the Swedish Foundation for Strategic Research to Sahlgrenska Centre for Cardiovascular and Metabolic Research, and The Health and Medical Care Committee of the Region Västra Götaland.

The authors' responsibilities were as follows: conception and design of the study and obtaining funding, L. G., J. T., J. K. and A. K. L.; acquisition of data, L. G.; analysis and interpretation of data, and writing the manuscript, L. G., J. T., J. K. and A. K. L.

None of the authors had a personal or financial conflict of interest.

\section{References}

1. Gilden Tsai A \& Wadden TA (2006) The evolution of very-lowcalorie diets: an update and meta-analysis. Obesity (Silver Spring) 14, 1283-1293.

2. SCOOP-VLCD Task 7.3 (2002) Reports on tasks for scientific cooperation. Collection of data on products intended for use in very-low-calorie-diets. http://ec.europa.eu/food/fs/scoop/7.3_ en.pdf (accessed 5 June 2009).

3. Bryson JM, King SE, Burns CM, et al. (1996) Changes in glucose and lipid metabolism following weight loss produced by a very low calorie diet in obese subjects. Int J Obes Relat Metab Disord 20, 338-345.

4. Colles SL, Dixon JB, Marks P, et al. (2006) Preoperative weight loss with a very-low-energy diet: quantitation of changes in liver and abdominal fat by serial imaging. Am J Clin Nutr 84, 304-311.

5. Martin CK, O'Neil PM \& Pawlow L (2006) Changes in food cravings during low-calorie and very-low-calorie diets. Obesity (Silver Spring) 14, 115-121.

6. Heymsfield SB, Harp JB, Reitman ML, et al. (2007) Why do obese patients not lose more weight when treated with low-calorie diets? A mechanistic perspective. Am J Clin Nutr 85, 346-354.

7. Wadden TA, Foster GD, Wang J, et al. (1992) Clinical correlates of short- and long-term weight loss. Am J Clin Nutr 56, 271S-274S

8. Anderson JW, Konz EC, Frederich RC, et al. (2001) Long-term weight-loss maintenance: a meta-analysis of US studies. Am J Clin Nutr 74, 579-584.
9. Stotland SC \& Larocque M (2005) Early treatment response as a predictor of ongoing weight loss in obesity treatment. $\mathrm{Br} J$ Health Psychol 10, 601-614.

10. Elfhag K \& Rossner S (2005) Who succeeds in maintaining weight loss? A conceptual review of factors associated with weight loss maintenance and weight regain. Obes Rev 6, 67-85.

11. Lantz H, Peltonen M, Agren L, et al. (2003) Intermittent versus on-demand use of a very low calorie diet: a randomized 2-year clinical trial. J Intern Med 253, 463-471.

12. Torgerson JS, Lissner L, Lindroos AK, et al. (1997) VLCD plus dietary and behavioural support versus support alone in the treatment of severe obesity. A randomised two-year clinical trial. Int J Obes Relat Metab Disord 21, 987-994.

13. Kaukua J, Pekkarinen T, Sane T, et al. (2003) Health-related quality of life in obese outpatients losing weight with verylow-energy diet and behaviour modification - a 2-y follow-up study. Int J Obes Relat Metab Disord 27, 1233-1241.

14. Lejeune MP, Kovacs EM \& Westerterp-Plantenga MS (2003) Effect of capsaicin on substrate oxidation and weight maintenance after modest body-weight loss in human subjects. $\mathrm{Br} J$ Nutr 90, 651-659.

15. Kovacs EM, Lejeune MP, Nijs I, et al. (2004) Effects of green tea on weight maintenance after body-weight loss. Br J Nutr 91, 431-437.

16. Pasman WJ, Westerterp-Plantenga MS \& Saris WH (1997) The effectiveness of long-term supplementation of carbohydrate, chromium, fibre and caffeine on weight maintenance. Int $J$ Obes Relat Metab Disord 21, 1143-1151.

17. Svendsen M, Rissanen A, Richelsen B, et al. (2008) Effect of Orlistat on eating behavior among participants in a 3-year weight maintenance trial. Obesity (Silver Spring) 16, 327-333.

18. Mathus-Vliegen EM (2005) Long-term maintenance of weight loss with sibutramine in a GP setting following a specialist guided very-low-calorie diet: a double-blind, placebocontrolled, parallel group study. Eur J Clin Nutr 59, Suppl. 1, $\mathrm{S} 31-\mathrm{S} 39$

19. Fogelholm M, Kukkonen-Harjula K \& Oja P (1999) Eating control and physical activity as determinants of short-term weight maintenance after a very-low-calorie diet among obese women. Int J Obes Relat Metab Disord 23, 203-210.

20. Ryttig KR \& Rossner S (1995) Weight maintenance after a very low calorie diet (VLCD) weight reduction period and the effects of VLCD supplementation. A prospective, randomized, comparative, controlled long-term trial. J Intern Med 238, 299-306

21. Westerterp-Plantenga MS, Lejeune MP, Nijs I, et al. (2004) High protein intake sustains weight maintenance after body weight loss in humans. Int J Obes Relat Metab Disord 28, $57-64$.

22. Erondu N, Wadden T, Gantz I, et al. (2007) Effect of NPY5R antagonist MK-0557 on weight regain after very-low-calorie diet-induced weight loss. Obesity (Silver Spring) 15, 895-905.

23. Torgerson JS, Agren L \& Sjöström L (1999) Effects on body weight of strict or liberal adherence to an initial period of VLCD treatment. A randomised, one-year clinical trial of obese subjects. Int J Obes Relat Metab Disord 23, 190-197.

24. LeCheminant JD, Jacobsen DJ, Hall MA, et al. (2005) A comparison of meal replacements and medication in weight maintenance after weight loss. J Am Coll Nutr 24, 347-353.

25. Foster GD, Wadden TA, Peterson FJ, et al. (1992) A controlled comparison of three very-low-calorie diets: effects on weight, body composition, and symptoms. Am J Clin Nutr 55, $811-817$

26. Wadden TA, Bartlett S, Letizia KA, et al. (1992) Relationship of dieting history to resting metabolic rate, body composition, eating behavior, and subsequent weight loss. Am J Clin Nutr 56, 203S-208S. 
27. Raymond NC, de Zwaan M, Mitchell JE, et al. (2002) Effect of a very low calorie diet on the diagnostic category of individuals with binge eating disorder. Int J Eat Disord 31, 49-56.

28. Harris JA \& Benedict FG (1912) A Biometric Study of Basal Metabolism in Man. Washington, DC: The Carnegie Institute.

29. Livsmedelsverket (1997) Svenska Näringsrekommendationer (Swedish Nutrition Recommendations). Uppsala, Sweden: Livsmedelsverket.

30. National Cholesterol Education Program (NCEP) Expert Panel on Detection, Evaluation, and Treatment of High Blood Cholesterol in Adults (Adult Treatment Panel III) (2002) Third Report of the National Cholesterol Education Program (NCEP) Expert Panel on Detection, Evaluation, and Treatment of High Blood Cholesterol in Adults (Adult Treatment Panel III) final report. Circulation 106, 3143-3421.

31. Karlsson J, Persson LO, Sjöström L, et al. (2000) Psychometric properties and factor structure of the Three-Factor Eating Questionnaire (TFEQ) in obese men and women. Results from the Swedish Obese Subjects (SOS) study. Int J Obes Relat Metab Disord 24, 1715-1725.
32. Tholin S, Rasmussen F, Tynelius P, et al. (2005) Genetic and environmental influences on eating behavior: the Swedish Young Male Twins Study. Am J Clin Nutr 81, 564-569.

33. McGuire MT, Jeffery RW, French SA, et al. (2001) The relationship between restraint and weight and weightrelated behaviors among individuals in a community weight gain prevention trial. Int $J$ Obes Relat Metab Disord 25, 574-580.

34. Borg P, Fogelholm M \& Kukkonen-Harjula K (2004) Food selection and eating behaviour during weight maintenance intervention and 2-y follow-up in obese men. Int J Obes Relat Metab Disord 28, 1548-1554

35. Clark AM, Thornley B, Tomlinson L, et al. (1998) Weight loss in obese infertile women results in improvement in reproductive outcome for all forms of fertility treatment. Hum Reprod 13, $1502-1505$.

36. Lindroos AK, Lissner L \& Sjöström L (1993) Validity and reproducibility of a self-administered dietary questionnaire in obese and non-obese subjects. Eur J Clin Nutr 47, $461-481$. 